\title{
miR-130a alleviates neuronal apoptosis and changes in expression of Bcl-2/Bax and caspase-3 in cerebral infarction rats through PTEN/PI3K/Akt signaling pathway
}

\author{
YINMING WANG*, JUNQUAN GU*, LINLIN HU, LIANG KONG, TINGGANG WANG, \\ MEIQI DI, CHAOSHENG LI and SHUHUA GUI
}

Department of Neurology, The Third People's Hospital of Wuxi, Wuxi, Jiangsu 214041, P.R. China

Received October 8, 2019; Accepted December 4, 2019

DOI: $10.3892 / \mathrm{etm} .2020 .8415$

\begin{abstract}
Effect of micro ribonucleic acid (miR)-130a on neuronal apoptosis in rats with cerebral infarction (CI) was studied to explore whether phosphatase and tensin homolog deleted on chromosome ten (PTEN)/phosphatidylinositol 3-hydroxy kinase (PI3K)/protein kinase B (Akt) is involved in the regulation of neuronal apoptosis. Thirty-six Sprague-Dawley (SD) rats were randomly divided into blank control group, model group and miR-130a low-expression group. miR-130a was determined by quantitative polymerase chain reaction (qPCR), the content of tumor necrosis factor- $\alpha$ (TNF- $\alpha$ ), interleukin-6 (IL-6) and IL-10 was detected using the enzyme-linked immunosorbent assay (ELISA) kits, and the neuronal apoptosis level in each group was determined through terminal deoxynucleotidyl transferase-mediated dUTP nick end labeling (TUNEL) staining. The neurobehavioral score was significantly lower in model group than that in blank control group $(\mathrm{P}<0.01)$, while it was significantly higher in miR-130a low-expression group than that in model group $(\mathrm{P}<0.01)$. Compared with blank control group, the model group had obviously increased content of TNF- $\alpha$ and IL-6 $(\mathrm{P}<0.01)$, decreased content of IL-10 $(\mathrm{P}<0.01)$, more apoptotic neurons $(\mathrm{P}<0.01)$, higher expression of caspase-3 $(\mathrm{P}<0.01)$, and obviously lower Bcl-2/Bax $(\mathrm{P}<0.01)$. Moreover, expression of phosphorylated (p)-PTEN, PI3K and p-Akt in brain tissues was remarkably lower in the model group than those in the blank control group $(\mathrm{P}<0.01)$. The expression level of miR-130a in brain tissues of CI rats is significantly increased. miR-130a promotes the release of inflammatory factors and facilitates
\end{abstract}

Correspondence to: Dr Shuhua Gui, Department of Neurology, The Third People's Hospital of Wuxi, 585 Xinyuanbei Road, Liangxi, Wuxi, Jiangsu 214041, P.R. China

E-mail: ikdps827@163.com

*Contributed equally

Key words: miR-130a, cerebral infarction, PTEN/PI3K/Akt signaling pathway, apoptosis neuronal apoptosis through suppressing the PTEN/PI3K/Akt signaling pathway.

\section{Introduction}

Stroke is one of the diseases seriously endangering human life health. According to the epidemiological survey of the World Health Organization, the number of deaths due to stroke ranks 2nd among all major diseases, and stroke is characterized by acute onset, a high mortality rate and many complications. China has gradually stepped into the stage of population aging, and stroke has become the first major cause of deaths $(1,2)$. Stroke has high mortality and disability rates, and edema will occur rapidly with space occupation after cerebral infarction (CI), leading to intracranial hypertension and cerebral hernia, and resulting in death (3). Currently, imaging is an important technique for diagnosing and evaluating the severity of CI, and there is a large amount of research evidence that the volume of cerebral edema after CI is closely related to the treatment and prognosis of CI patients (4-6). However, the volume of edema cannot be detected by imaging until the disease occurs, causing a lag effect, so it is vitally important to search for new indexes for the diagnosis and prediction of the therapeutic effect and prognosis of CI patients.

A large amount of research evidence has confirmed that micro ribonucleic acids (miRNAs) are involved in regulating various pathophysiological processes, which are closely related to cell apoptosis, proliferation and individual development (7,8). Tan et al (9) analyzed the tissue samples of stroke patients using bioinformatics, and found that stroke will cause significant changes in the expression of miRNAs. Ding et al (10) found that the expression level of miR-130a significantly increases after ischemia-reperfusion, and it is closely related to the therapeutic effect and prognosis of patients. The role of phosphatase and tensin homolog deleted on chromosome ten (PTEN), as a cancer suppressor gene with phosphatase activity, in brain injury has gradually received attention, and strong research evidence exists to support the close correlation of PTEN with neuronal proliferation and apoptosis. Furthermore, PTEN can also activate the downstream phosphatidylinositol 3-hydroxy kinase (PI3K)/protein kinase $\mathrm{B}$ (Akt) signaling pathway, and participate in the stress 
induced by cerebral ischemia-reperfusion (11). However, there is scarce literature on the effects of miR-130a on cerebral ischemia and PTEN/PI3K/Akt signaling pathway at present. Therefore, in this study, the rat model of CI was established to evaluate the effect of miR-130a on neuronal injury in $\mathrm{CI}$ rats and whether the PTEN/PI3K/Akt signaling pathway is involved in the regulation of this process.

\section{Materials and methods}

Laboratory animals and grouping. Thirty-six male Sprague-Dawley (SD) rats (250-280 g) were adaptively fed in a specific pathogen-free environment for 1 week under the temperature of $23 \pm 2^{\circ} \mathrm{C}$, humidity of $45 \pm 5 \%$ and regular circadian rhythm and had free access to food and water. At $12 \mathrm{~h}$ before the experiment, the rats were deprived of food, not water.

The above rats were randomly divided into blank control group ( $\mathrm{n}=12)$, model group $(\mathrm{n}=12)$ and miR-130a low-expression group $(n=12)$. No treatment was performed in blank control group, and the CI model was established in the model group and miR-130a low-expression group. miR-130a inhibitor negative control was injected into the lateral ventricle of rats before modeling in the model group, while miR-130a inhibitors were injected into the lateral ventricle of rats before modeling in the miR-130a low-expression group. This research was approved by the Animal Ethics Committee of the Animal Center of the Third People's Hospital of Wuxi (Wuxi, China).

Injection of miR-130a inhibitors into lateral ventricle. The miR-130a inhibitors and miR-130a inhibitor negative control were purchased from Shanghai GenePharma Co., Ltd. The lyophilized powder was diluted with RNase-free $\mathrm{H}_{2} \mathrm{O}$ to a concentration of $200 \mu \mathrm{mol} / 1$ and incubated at room temperature for $5 \mathrm{~min}$. Then it was gently mixed with Lipofectamine 2000 (Invitrogen; Thermo Fisher Scientific, Inc.) diluted with serum-free Dulbecco's modified Eagle's medium (DMEM) (Gibco; Thermo Fisher Scientific, Inc.) and incubated at room temperature for $5 \mathrm{~min}$. Lipofectamine $^{\mathrm{TM}} 2000$ was mixed with miR-130a inhibitors and miR-130a inhibitor negative control, respectively, incubated at room temperature for $20 \mathrm{~min}$ and stored for later use. After the rats were anesthetized with $10 \%$ chloral hydrate via intraperitoneal injection at a dose of $300-350 \mathrm{mg} / \mathrm{kg}$, the head skin was cut to expose the anterior fontanel, and the rats were fixed on a stereotaxic apparatus. No rat exhibited signs of peritonitis after the administration of $10 \%$ chloral hydrate. With the anterior fontanel as the original point, the parameters of the stereotaxic apparatus were adjusted to: $0.8 \mathrm{~mm}(\mathrm{P}), 1.5 \mathrm{~mm}(\mathrm{R})$, and $4.5 \mathrm{~mm}(\mathrm{~V})$. Then the skull was drilled, from which the miR-130a inhibitors and miR-130a inhibitor negative control were injected. After $60 \mathrm{~min}$, the rat model of CI was established.

Establishment of CI model. After anesthesia via 10\% chloral hydrate by intraperitoneal injection at a dose of $300-350 \mathrm{mg} / \mathrm{kg}$, the rats were fixed on a plate in a supine position, and the neck hair was shaved off. No rat exhibited signs of peritonitis after the administration of $10 \%$ chloral hydrate. A longitudinal incision was made in the middle-right line of the neck, and the subcutaneous tissues were separated using surgical instruments. The distal external carotid artery was ligated with the thread, and the common carotid artery and internal carotid artery were clamped with artery clamps. A small incision was made in the external carotid artery, from which the suture was inserted. Then the artery clamps in the internal carotid artery were released, and the suture was reversely inserted into the internal carotid artery to block the blood supply of the middle cerebral artery. After that, the rats were fed in separate cages until resuscitation, and the water drinking status and wounds were observed. In blank control group, the suture was not inserted, and the remaining operations were the same as those in the model group.

Evaluation of behavioral changes in rats via behavioral experiments. At 3 days after modeling, the behavioral changes of rats in each group were evaluated using the following three behavioral experiments: i) Rotation response: 0 points, no rotation; 2 points, rotation towards the affected side; and 4 points, spontaneous rotation or death. ii) Forepaw grip: The rats in each group were suspended using the wire rope at $60 \mathrm{~cm}$ above the ground, with the hind limbs away from the ground. The score was given based on the suspension time: 0 point, $>20 \mathrm{sec}$; 2 points, $<10 \mathrm{sec}$; 3 points, the rats fell immediately after catching the rope; and 4 points, the rats could not catch the rope. iii) Crossbar walking: The rats were put on a crossbar ( $2 \mathrm{~cm}$ wide and $60 \mathrm{~cm}$ long), and their passage was recorded. 0 point: The rats went across the crossbar, 1 point, the rats fell from the crossbar after $30 \mathrm{~cm} ; 2$ points, the rats fell from the crossbar before $30 \mathrm{~cm} ; 3$ points, the rats lay on the crossbar and did not walk; 4 points, the rats fell immediately. After behavioral experiments, the rats in each group were sacrificed immediately, and the whole brain was taken out. The tissues around the hemorrhagic hemisphere were isolated and stored at $-80^{\circ} \mathrm{C}$ for later use.

Detection of miR-130a expression level in brain tissues using quantitative polymerase chain reaction ( $q P C R)$. At 3 days after modeling, the rats in each group were sacrificed immediately, and the tissues around the hemorrhagic hemisphere were isolated and weighed. Then the tissues were added with TRIzol reagent (Invitrogen; Thermo Fisher Scientific, Inc.) at a mass/volume ratio of $100 \mathrm{mg} / \mathrm{ml}$, homogenized using a homogenizer and placed at room temperature for $10 \mathrm{~min}$. Chloroform $(200 \mu \mathrm{l})$ was added and the mixture was placed at room temperature for $5 \mathrm{~min}$, followed by centrifugation at $10,500 \mathrm{x} \mathrm{g}$ and $4^{\circ} \mathrm{C}$ for $10 \mathrm{~min}$. Then the supernatant was taken into new centrifuge tubes, and the total RNA was extracted according to instructions of the RNA extraction kit (Vazyme). The absorbance $(\mathrm{A})_{260} / \mathrm{A}_{280}$ and optical density (OD) value of total RNA were determined, the quality and concentration of RNA were evaluated, and RNA was stored at $-30^{\circ} \mathrm{C}$ for later use. The reverse transcription system was prepared in strict accordance with the instructions of the reverse transcription kit (Vazyme), and the PCR conditions were: at $37^{\circ} \mathrm{C}$ for $15 \mathrm{~min}$ and at $85^{\circ} \mathrm{C}$ for $5 \mathrm{~min}$. Moreover, the qPCR system was prepared for amplification, as follows: pre-denaturation at $95^{\circ} \mathrm{C}$ for $30 \mathrm{sec}$; PCR: at $95^{\circ} \mathrm{C}$ for $5 \mathrm{sec}$, at $60^{\circ} \mathrm{C}$ for $30 \mathrm{sec}$, for a total of 40 cycles. With $\beta$-actin as an internal reference, the primers were synthesized by Invitrogen; Thermo Fisher Scientific, 
Table I. Primers used in this study.

\begin{tabular}{lll}
\hline Gene & \multicolumn{1}{c}{ Forward primer } & \multicolumn{1}{c}{ Reverse primer } \\
\hline miR-130a & 5'-CAGTGCAATGTTAAAAGGGCAT-3' & 5'-CTCGCTTCGGCAGCACA-3' \\
$\beta$-actin & 5'-AACCGTCGGGGACGGAT-3' & 5'-TGGCGATCAGACGCAGGTC-3'
\end{tabular}

Inc. (Table I). The relative expression level of miR-130a was calculated using $2^{-\Delta \Delta \mathrm{Ct}}$, and expressed as miR-130a/ $\beta$-actin.

Determination of content of inflammatory factors. At 3 days after modeling, the rats in each group were sacrificed immediately, and the tissues around the hemorrhagic hemisphere were isolated. Then inflammatory factors in brain tissues were measured using the enzyme-linked immunosorbent assay (ELISA) kits of tumor necrosis factor- $\alpha$ (TNF- $\alpha$ ), interleukin-6 (IL-6) and IL-10 (Wuhan Boster Biological Technology Co., Ltd.) in strict accordance with the instructions, and recorded in detail.

Determination of the number of apoptotic cells using terminal deoxynucleotidyl transferase-mediated dUTP nick end labeling (TUNEL) staining. At 3 days after modeling, the rats in each group were sacrificed immediately, and the tissues around the hemorrhagic hemisphere were isolated and prepared into paraffin sections. The neuronal apoptosis level of brain tissues in each group was detected through TUNEL staining: After deparaffinization, the paraffin sections were treated with $3 \%$ hydrogen peroxide for $10 \mathrm{~min}$, rinsed with phosphate-buffered saline (PBS) 3 times, dropwise added with proteinase $\mathrm{K}$ solution, and incubated in a wet box at $37^{\circ} \mathrm{C}$ for $10 \mathrm{~min}$. After sections were rinsed with PBS 3 times, they were dropwise added with mixed solution of TdT and DIG-d-UTP, and incubated in the wet box at $4^{\circ} \mathrm{C}$ for $2 \mathrm{~h}$. After sections were rinsed with PBS 3 times, they were sealed with $40 \mu \mathrm{l}$ of blocking buffer at room temperature for $30 \mathrm{~min}$, dropwise added with antibodies $(1: 100)$ and incubated in a wet box at $37^{\circ} \mathrm{C}$ for $30 \mathrm{~min}$. After sections were rinsed with PBS 3 times, they were dropwise added with the SABC-FITC secondary antibody (1:100) for incubation in the wet box at $37^{\circ} \mathrm{C}$ for $30 \mathrm{~min}$, and rinsed again with PBS 3 times. Finally, the sections were added dropwise with anti-fluorescence quenching blocking buffer, and observed and photographed under a confocal fluorescence microscope. The number of TUNEL-positive cells in the hemorrhagic hemisphere was recorded to evaluate the neuronal apoptosis level (the TUNEL-positive cells displayed yellow green fluorescence).

Detection of related protein expression through western blotting. At 3 days after modeling, the rats in each group were sacrificed immediately, and the tissues around the hemorrhagic hemisphere were isolated, weighed and added with radioimmunoprecipitation assay (RIPA) lysis buffer (Beijing TDY Biotech Co., Ltd.) at a mass/volume ratio of $100 \mathrm{mg} / \mathrm{ml}$, as well as $1 \%$ phosphatase inhibitor and $1 \%$ protease inhibitor, followed by homogenization using the homogenizer, standing at room temperature for $10 \mathrm{~min}$ and centrifugation at $10,000 \times \mathrm{g}$ and $4^{\circ} \mathrm{C}$ for $10 \mathrm{~min}$. Then the supernatant was taken into new centrifuge tubes as the total protein, and the total protein was quantified using the bicinchoninic acid (BCA) protein assay kit (Pierce; Thermo Fisher Scientific, Inc.).

The total protein in each group was diluted with loading buffer until the same concentration, and boiled for $10 \mathrm{~min}$ for inactivation. After the sodium dodecyl sulphate-polyacrylamide gel electrophoresis (SDS-PAGE) gel was prepared, an equal volume of protein samples was added for electrophoresis under $80 \mathrm{~V}$ until the blue band reached the end of separation gel. After that, the protein was transferred onto a polyvinylidene fluoride (PVDF) membranes (Millipore) using the wet method under the constant pressure of $100 \mathrm{~V}$ for $90 \mathrm{~min}$. Then the protein band was sealed with freshly-prepared 5\% skim milk powder for $1 \mathrm{~h}$, and the target band was cut and incubated with the primary antibodies of caspase-3 (1:1,000), B-cell lymphoma-2 (Bcl-2) associated X protein (Bax) $(1: 1,000), \mathrm{Bcl}-2(1: 1,000)$, phosphorylated (p)-PTEN (1:1,000), PTEN (1:1,000), PI3K (1:1,000), p-Akt $(1: 1,000)$, Akt $(1: 1,000)$ and GAPDH (internal reference antibody, 1:1,000) (all from Cell Signaling Technology, Inc.) at $4^{\circ} \mathrm{C}$ overnight. The band was washed with Tris-buffered saline with Tween-20 (TBST) 3 times ( $5 \mathrm{~min} / \mathrm{time}$ ), incubated again with the horseradish peroxidase-labeled goat anti-rabbit secondary antibody (1:5,000; Shanghai Yihyson Biological Co., Ltd.) at room temperature for $1 \mathrm{~h}$ and washed again with TBST 3 times ( $5 \mathrm{~min} / \mathrm{time}$ ). Then an appropriate amount of electrochemiluminescence (ECL) solution was added to each band, followed by color development using the developing instrument. The results were processed using ImageJ, and the expression level of each protein was calculated.

Statistical analysis. The data in this study were expressed as mean \pm standard deviation. Statistical Product and Service Solutions (SPSS) 22.0 software (IBM, Corp.) was used for the data processing. The t-test was used for analyzing measurement data. Differences between two groups were analyzed by using the Student's t-test. Comparison between multiple groups was done using One-way ANOVA test followed by Post Hoc Test (Least Significant Difference). $\mathrm{P}<0.05$ indicates that the difference was statistically significant.

\section{Results}

Neurological function of rats. The neurological function of rats in each group was evaluated using behavioral experiments. As shown in Fig. 1, the scores in rotation test, traction test and crossbar test were significantly higher in the model group than those in the blank control group $(\mathrm{P}<0.01, \mathrm{P}<0.01, \mathrm{P}<0.01)$, while they significantly declined after intervention with miR-130a inhibitors $(\mathrm{P}<0.01, \mathrm{P}<0.01, \mathrm{P}<0.01$, respectively). 
A

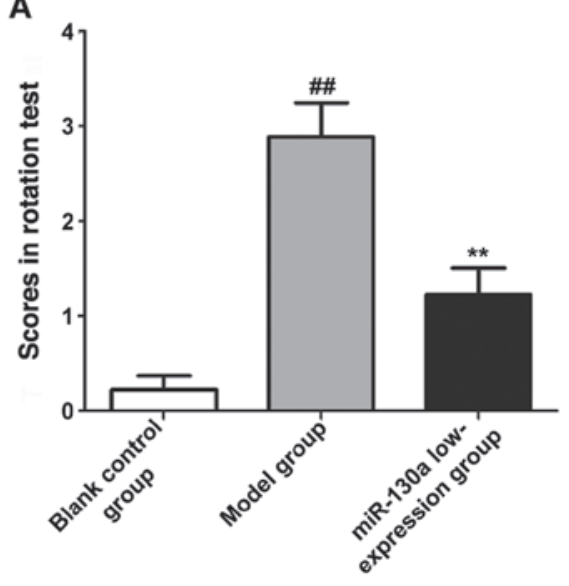

B

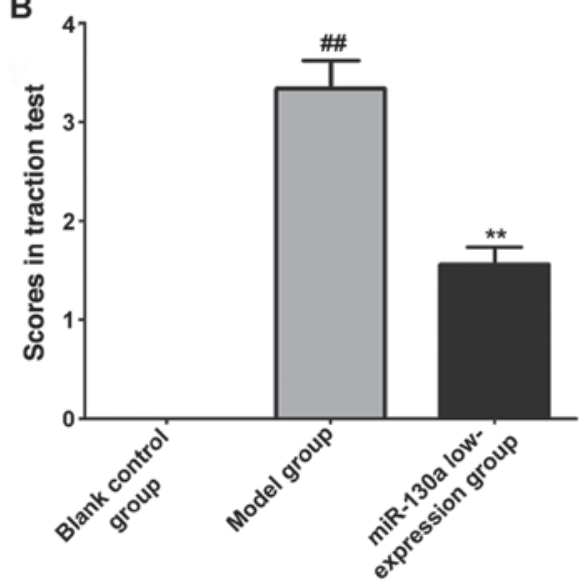

C

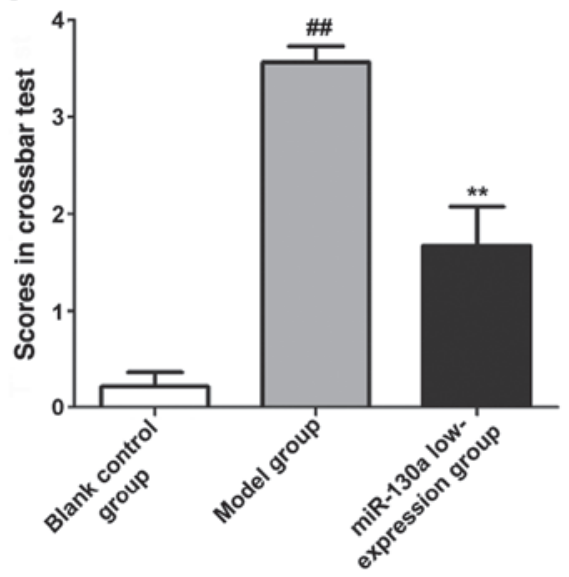

Figure 1. Neurological function of rats in each group evaluated using behavioral experiments. (A) Rotation test, (B) traction test, and (C) crossbar test. The scores in rotation test, traction test and crossbar test are significantly higher in model group than those in blank control group, while they are significantly lower in miR-130a low-expression group than those in model group. ${ }^{\# \#} \mathrm{P}<0.01$ vs. blank control group; ${ }^{* *} \mathrm{P}<0.01$ vs. model group.

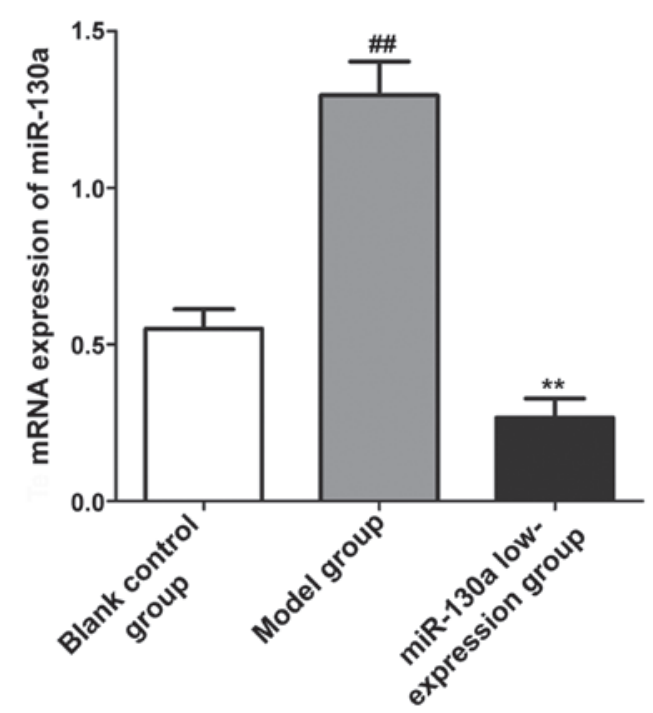

Figure 2. Expression of miR-130a in brain tissues in each group detected using qPCR. The expression level of miR-130a in brain tissues is obviously higher in model group than that in blank control group, while it is obviously lower in miR-130a low-expression group than that in model group. ${ }^{\# \#} \mathrm{P}<0.01$ vs. blank control group; ${ }^{* *} \mathrm{P}<0.01$ vs. model group.

Expression of miR-130a in brain tissues. The relative expression level of miR-130a in CI tissues in each group was detected via qPCR. As shown in Fig. 2, the expression level of miR-130a in CI tissues was obviously increased in model group $(\mathrm{P}<0.01)$, while it obviously declined in miR-130a low-expression group after intervention with miR-130a inhibitors $(\mathrm{P}<0.01)$.

Content of inflammatory factors in brain tissues. The content of inflammatory factors in CI tissues in each group was determined using the ELISA kits. The results showed that compared with blank control group, model group had evidently increased content of IL- 6 and TNF- $\alpha$ in brain tissues $(\mathrm{P}<0.01)$ and evidently decreased content of IL-10 $(\mathrm{P}<0.01)$. After intervention with miR-130a inhibitors, the content of IL-6 and TNF- $\alpha$ in brain tissues evidently declined $(\mathrm{P}<0.01)$ and that of IL-10 was evidently increased $(\mathrm{P}<0.01)$ in miR-130a low-expression group (Fig. 3).

Neuronal injury in brain tissues. The neuronal apoptosis level of CI tissues in each group was detected through TUNEL staining, and the number of TUNEL-positive cells was counted to indicate the number of apoptotic neurons. It was found that the number of TUNEL-positive cells was obviously larger in model group than that in blank control group $(\mathrm{P}<0.01)$, while it was obviously smaller in miR-130a low-expression group than that in model group $(\mathrm{P}<0.01)$ (Fig. 4).

Expression levels of apoptosis proteins in brain tissues. The expression levels of apoptosis-related proteins in CI tissues in each group were detected using western blotting. The results revealed that compared with blank control group, model group had a remarkably lower expression of $\mathrm{Bcl}-2 / \mathrm{Bax}(\mathrm{P}<0.01)$ and a remarkably higher expression of caspase- 3 in brain tissues $(\mathrm{P}<0.01)$. Compared with model group, miR-130a low-expression group had a remarkably higher expression of $\mathrm{Bcl}-2 / \mathrm{Bax}$ $(\mathrm{P}<0.01)$ and a remarkably lower expression of caspase-3 in brain tissues $(\mathrm{P}<0.01)$ (Fig. 5).

Expression levels of PTEN/PI3K/Akt pathway-related proteins in brain tissues. The expression levels of PTEN/PI3K/Akt signaling pathway-related proteins in CI tissues in each group were also detected using western blotting. As shown in Fig. 6, the expression of p-PTEN, PI3K and p-Akt in brain tissues was remarkably lower in model group than those in blank control group $(\mathrm{P}<0.01)$, while inhibiting the expression of miR-130a remarkably increased the expression of p-PTEN, PI3K and $\mathrm{p}$-Akt in brain tissues in the model group $(\mathrm{P}<0.01)$.

\section{Discussion}

Hemorrhagic stroke is a serious disease leading to death, and a large amount of research evidence demonstrates that the volume of hematoma in CI and the complications of patients are important factors affecting prognosis, which can be used to evaluate the prognosis of CI patients (12). MiRNAs are a 
A

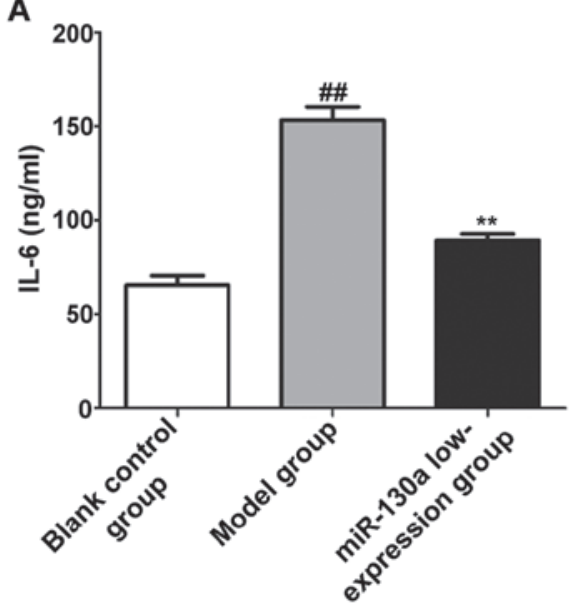

B

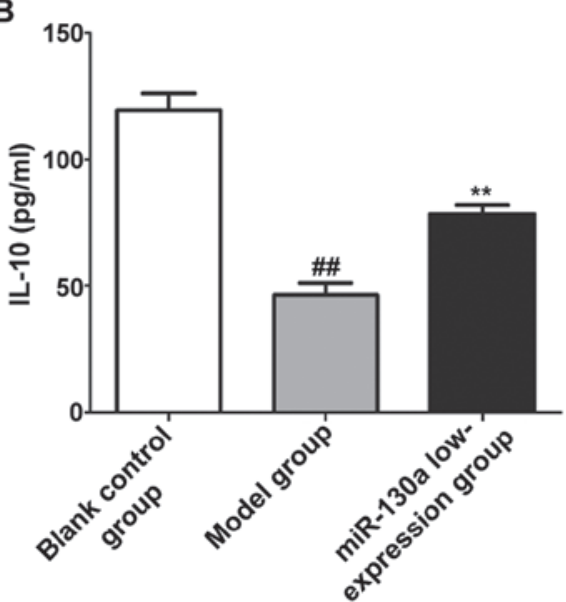

C

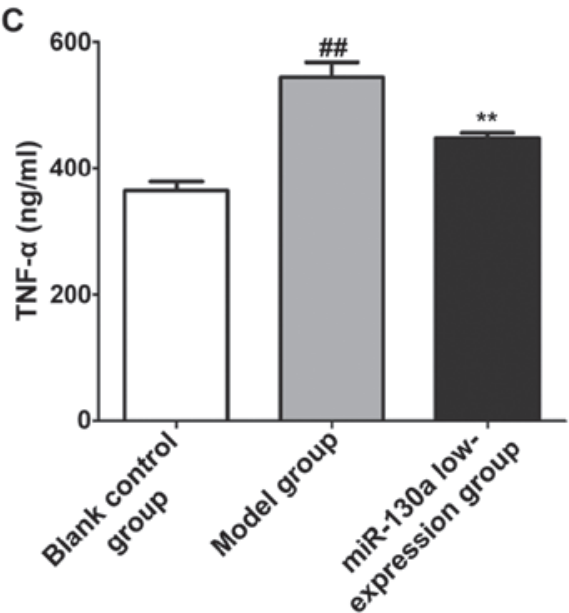

Figure 3. Content of inflammatory factors in brain tissues in each group. (A) Content of IL-6, (B) content of IL-10, and (C) content of TNF- $\alpha$. Compared with blank control group, model group has evidently increased content of IL- 6 and TNF- $\alpha$ in brain tissues and evidently decreased content of IL-10. ${ }^{\# \#} \mathrm{P}<0.01$ vs. blank control group; ${ }^{* *} \mathrm{P}<0.01$ vs. model group. IL, interleukin; TNF- $\alpha$, tumor necrosis factor- $\alpha$.
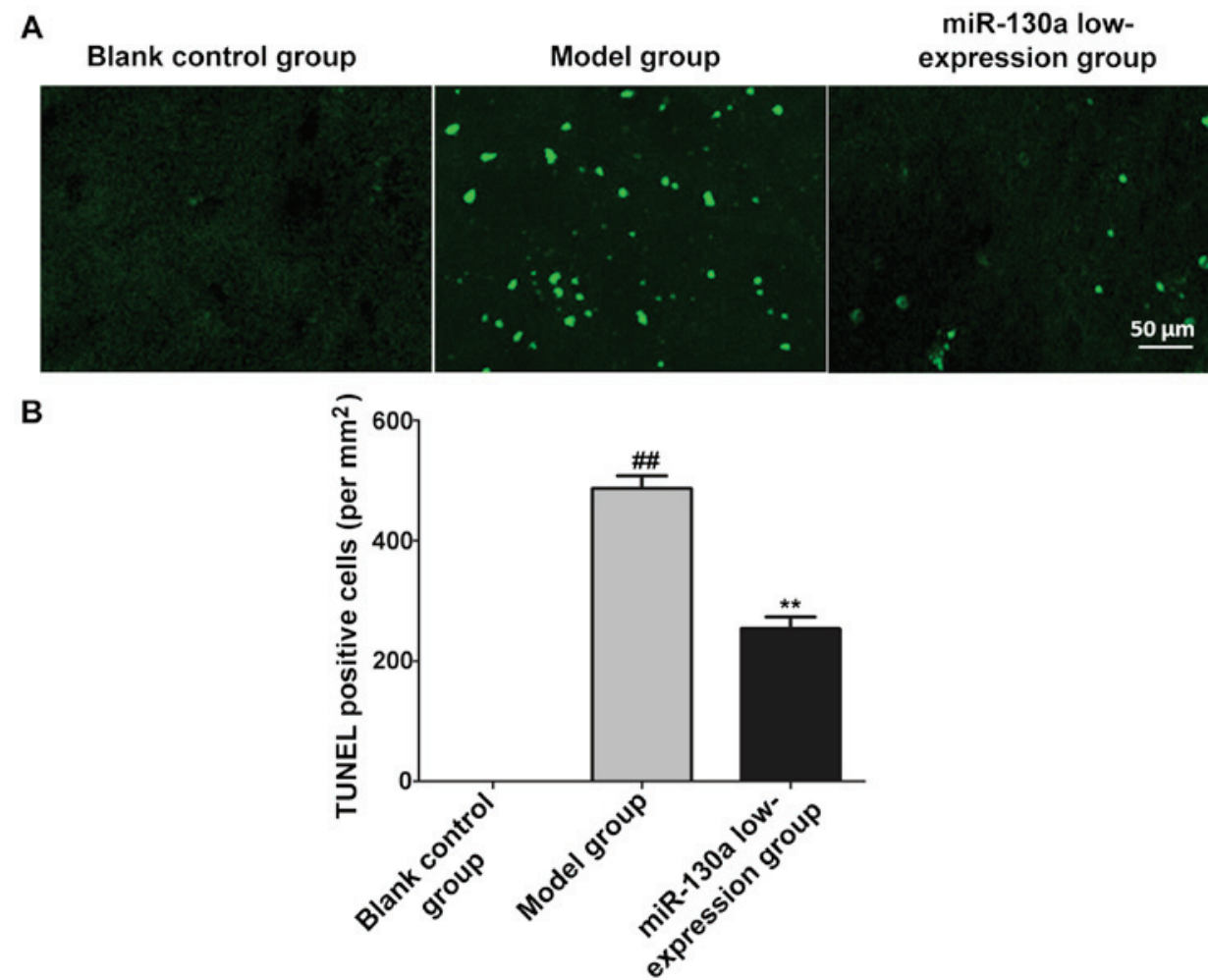

Figure 4. Neuronal apoptosis level in each group detected through TUNEL staining. (A) Micrograph, and (B) Statistical graph (scale bar, $50 \mu \mathrm{m}$ ). The number of TUNEL-positive cells in brain tissues is obviously larger in model group than that in blank control group and miR-130a low-expression group. ${ }^{\# \#} \mathrm{P}<0.01$ vs. blank control group; ${ }^{* *} \mathrm{P}<0.01$ vs. model group.

group of non-coding RNAs that play regulatory roles in vivo, and they widely exist in many eukaryotic organisms, which are closely related to the individual development, energy metabolism, cell proliferation and apoptosis (13). For example, Selvamani et al (14) found that such miRNAs as miR-21 and miR-181b are involved in the formation of cerebral edema, and they are important factors affecting the prognosis of stroke patients. miR-130a is an anti-angiogenic transcription factor. The study of Jiang et al (15) showed that the expression of miR-130a is positively proportional to the cerebral water content in rats with cerebral edema, suggesting that miR-130a may be associated with the formation of cerebral edema (16). Furthermore, Altintas et al (17) found that miR-130a also plays an important regulatory role in diabetic rats with transient $\mathrm{CI}$. In the present study, the rat model of CI was established. It was found that the expression level of miR-130a in CI tissues in model group was significantly increased, and the results of behavioral experiments revealed that there was severe neurological injury in model group. After intervention in the expression of miR-130a in brain tissues, the neurological function was effectively restored. The 
A

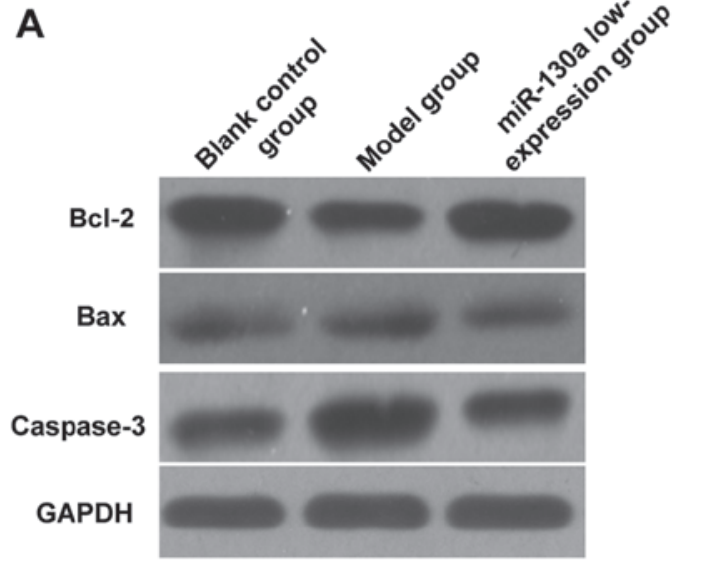

B

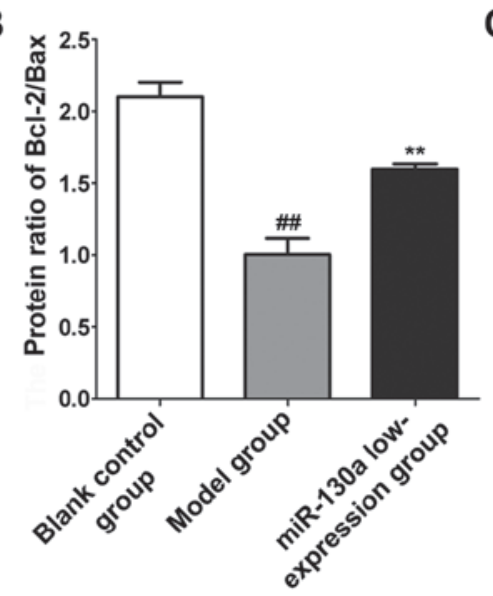

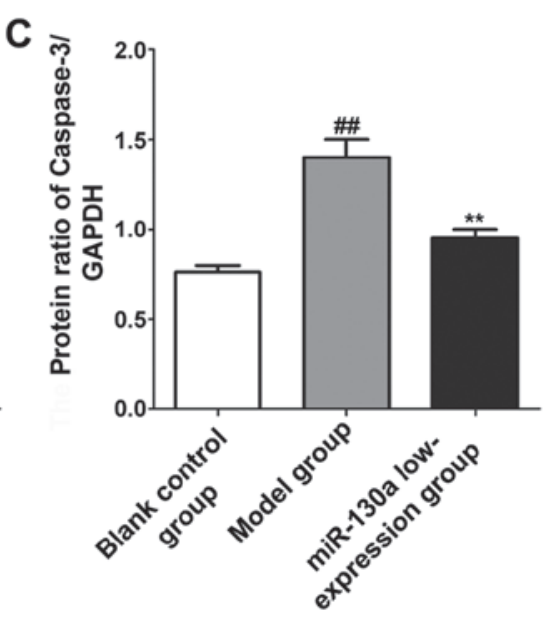

Figure 5. Expression levels of apoptosis-related proteins in CI tissues in each group. (A) Protein bands, (B) statistical graph of Bcl-2/Bax, and (C) statistical graph of caspase-3. The expression of Bcl-2/Bax in CI tissues in model group is remarkably lower than that in blank control group and miR-130a low-expression group. The expression of caspase-3 is remarkably higher in model group than that in blank control group and miR-130a low-expression group. ${ }^{\# \#} \mathrm{P}<0.01$ vs. blank control group; ${ }^{* *} \mathrm{P}<0.01$ vs. model group. $\mathrm{CI}$, cerebral infarction; Bcl-2, B-cell lymphoma-2; Bax, associated $\mathrm{X}$ protein.

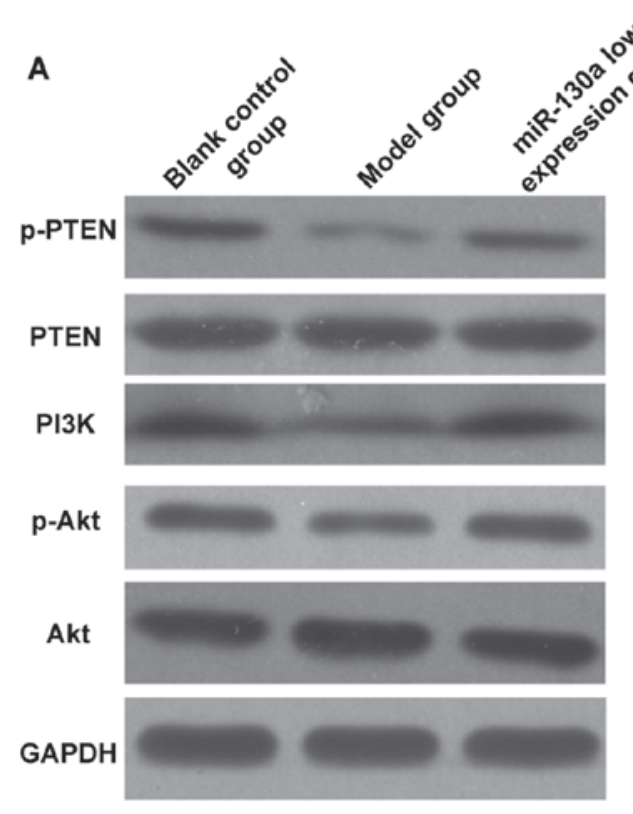

C

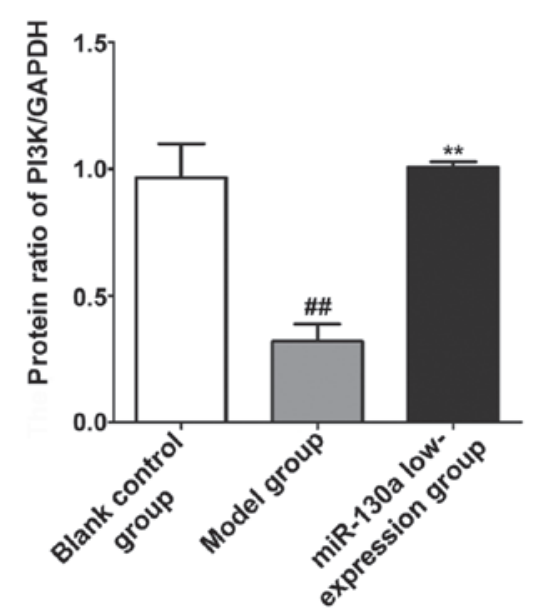

B
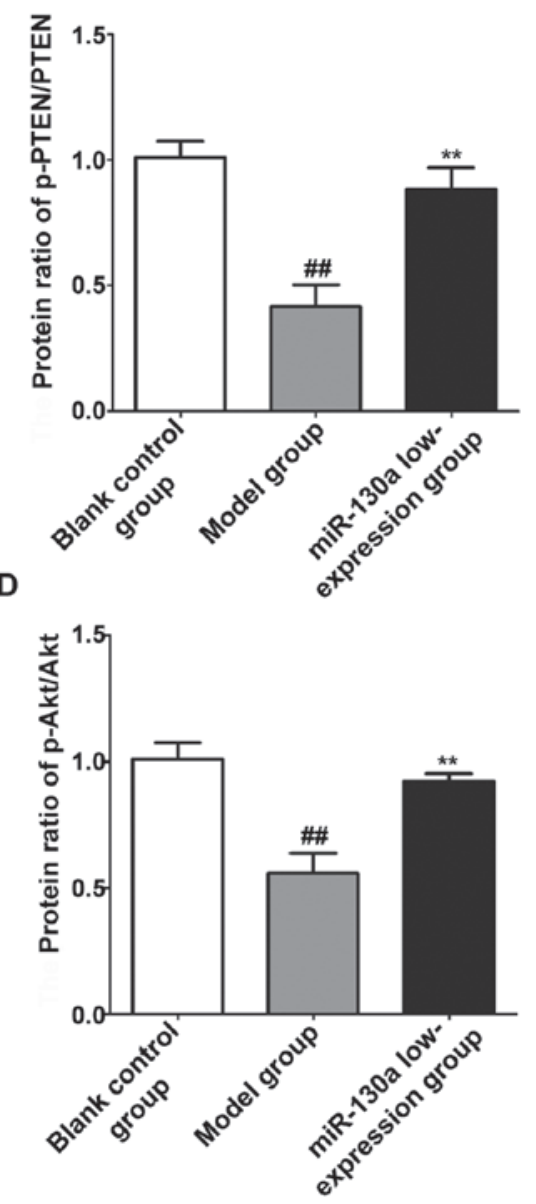

Figure 6. Expression levels of PTEN/PI3K/Akt pathway-related proteins in brain tissues in each group. (A) Protein bands, (B) expression level of p-PTEN, (C) expression level of PI3K, and (D) expression level of p-Akt. The expression of p-PTEN, PI3K and p-Akt in brain tissues in model group is significantly lower than those in blank control group and miR-130a low-expression group. ${ }^{\# \#} \mathrm{P}<0.01$ vs. blank control group; ${ }^{* *} \mathrm{P}<0.01$ vs. model group. PTEN, phosphatase and tensin homolog deleted on chromosome ten; PI3K, phosphatidylinositol 3-hydroxy kinase; Akt, protein kinase B. 
above findings indicate that the high expression of miR-130a may be closely related to brain injury in CI rats.

PTEN is a cancer suppressor gene with phosphatase activity, and it has been proven to be involved in cell invasion, migration and apoptosis (18). PI3K is the most important substrate for PTEN and an important messenger for cell proliferation, and PTEN can specifically act on PI3K to regulate the phosphorylation of Akt. p-Akt can directly or indirectly regulate the activity of apoptosis proteins, thereby disturbing cell proliferation and arresting cell cycle, and ultimately leading to apoptosis (19). After phosphorylation of PTEN, structural instability will be caused and phosphatase activity will be lost (20). In this study, it was found that after CI for 3 days, the expression level of p-PTEN in brain tissues in each group was obviously decreased, and the expression levels of PI3K and p-Akt in brain tissues of CI rats also obviously declined, suggesting that CI will reduce the phosphorylation level of PTEN in brain tissues, thus inhibiting PI3K/Akt and resulting in neuronal injury in rats. Moreover, in this study, the neuronal apoptosis level in brain tissues of CI rats was obviously increased, and the levels of pro-inflammatory factors IL- 6 and TNF- $\alpha$ significantly increased, while the level of anti-inflammatory factor IL-10 significantly declined. In addition, obvious changes were observed in the expression of apoptosis-related proteins Bcl-2/Bax and caspase-3. The above results highly demonstrate that the mechanism of CI may be that the phosphorylation of PTEN is suppressed, thereby inhibiting the activity of the PI3K/Akt signaling pathway, and causing neuronal injury and neuronal apoptosis. Besides, inflammatory factors are the basic substances causing inflammation, and their content evidently increases in brain tissues after CI, which can lead to the activation and infiltration of inflammatory cells in brain tissues, thereby producing a direct toxic effect on neurons in brain tissues and inducing cerebral edema (21).

In conclusion, CI can significantly raise the expression level of miR-130a in brain tissues in rats, thereby promoting the release of inflammatory factors and enhancing the neuronal apoptosis through inhibiting the PTEN/PI3K/Akt signaling pathway, ultimately causing typical symptoms of neurological injury in rats. The expression level of miR-130a may serve as an important index for evaluating the brain injury and prognosis of CI patients.

\section{Acknowledgements}

Not applicable.

\section{Funding}

No funding was received.

\section{Availability of data and materials}

All data generated or analyzed during this study are included in this published article.

\section{Authors' contributions}

YW, JG and SG designed the study and performed the experiments; LH, CL and LK established the animal models; JG and LK collected the data; TW and MD analyzed the data; YW, JG and CL prepared the manuscript. All authors read and approved the final manuscript.

\section{Ethics approval and consent to participate}

This research was approved by the Animal Ethics Committee of the Animal Center of the Third People's Hospital of Wuxi (Wuxi, China).

\section{Patient consent for publication}

Not applicable.

\section{Competing interests}

The authors declare they have no competing interests.

\section{References}

1. Lackland DT, Roccella EJ, Deutsch AF, Fornage M, George MG, Howard G, Kissela BM, Kittner SJ, Lichtman JH, Lisabeth LD, et al; American Heart Association Stroke Council; Council on Cardiovascular and Stroke Nursing; Council on Quality of Care and Outcomes Research; Council on Functional Genomics and Translational Biology: Factors influencing the decline in stroke mortality: A statement from the American Heart Association/American Stroke Association. Stroke 45: 315-353, 2014.

2. Bhaskar S, Stanwell P, Cordato D, Attia J and Levi C: Reperfusion therapy in acute ischemic stroke: Dawn of a new era? BMC Neurol 18: 8, 2018.

3. Liu D, Tang ZY, Hu ZJ, Li WW and Yuan WN: MiR-940 regulates angiogenesis after cerebral infarction through VEGF. Eur Rev Med Pharmacol Sci 22: 7899-7907, 2018.

4. Lin MP, Sanossian N and Liebeskind DS: Imaging of prehospital stroke therapeutics. Expert Rev Cardiovasc Ther 13: 1001-1015, 2015.

5. McCulloch L, Smith CJ and McColl BW: Adrenergic-mediated loss of splenic marginal zone B cells contributes to infection susceptibility after stroke. Nat Commun 8: 15051, 2017.

6. Gulli G, Rutten-Jacobs LC, Kalra L, Rudd AG, Wolfe CD and Markus HS: Differences in the distribution of stroke subtypes in a UK black stroke population - final results from the South London Ethnicity and Stroke Study. BMC Med 14: 77, 2016.

7. Weiss CN and Ito K: A Macro View of MicroRNAs: The discovery of microRNAs and their role in hematopoiesis and hematologic disease. Int Rev Cell Mol Biol 334: 99-175, 2017.

8. Soares RJ, Cagnin S, Chemello F, Silvestrin M, Musaro A, De Pitta C, Lanfranchi G and Sandri M: Involvement of microRNAs in the regulation of muscle wasting during catabolic conditions. J Biol Chem 289: 21909-21925, 2014.

9. Tan JR, Tan KS, Yong FL, Armugam A, Wang CW, Jeyaseelan K and Wong PT: MicroRNAs regulating cluster of differentiation 46 (CD46) in cardioembolic and non-cardioembolic stroke. PLoS One 12: e0172131, 2017.

10. Ding C, Chen SN, Macleod RAF, Drexler HG, Nagel S, Wu DP, Sun AN and Dai HP: MiR-130a is aberrantly overexpressed in adult acute myeloid leukemia with $\mathrm{t}(8 ; 21)$ and its suppression induces AML cell death. Ups J Med Sci 123: 19-27, 2018.

11. Zhu G, Zhang W, Liu Y and Wang S: miR 371b 5p inhibits endothelial cell apoptosis in monocrotaline induced pulmonary arterial hypertension via PTEN/PI3K/Akt signaling pathways. Mol Med Rep 18: 5489-5501, 2018.

12. Rothwell PM, Algra A, Chen Z, Diener HC, Norrving B and Mehta Z: Effects of aspirin on risk and severity of early recurrent stroke after transient ischaemic attack and ischaemic stroke: Time-course analysis of randomised trials. Lancet 388: 365-375, 2016.

13. Lamontagne J, Steel LF and Bouchard MJ: Hepatitis B virus and microRNAs: Complex interactions affecting hepatitis $B$ virus replication and hepatitis $B$ virus-associated diseases. World J Gastroenterol 21: 7375-7399, 2015. 
14. Selvamani A, Williams MH, Miranda RC and Sohrabji F Circulating miRNA profiles provide a biomarker for severity of stroke outcomes associated with age and sex in a rat model. Clin Sci (Lond) 127: 77-89, 2014.

15. Jiang H, Yu WW, Wang LL and Peng Y: miR-130a acts as a potential diagnostic biomarker and promotes gastric cancer migration, invasion and proliferation by targeting RUNX3. Oncol Rep 34: 1153-1161, 2015.

16. Zhang Y, Chen M, Qiu Z, Hu K, McGee W, Chen X, Liu J, Zhu L and Wu JY: MiR-130a regulates neurite outgrowth and dendritic spine density by targeting MeCP2. Protein Cell 7: 489-500, 2016.

17. Altintas O, Ozgen Altintas M, Kumas $M$ and Asil T: Neuroprotective effect of ischemic preconditioning via modulating the expression of cerebral miRNAs against transient cerebral ischemia in diabetic rats. Neurol Res 38: 1003-1011, 2016.

18. Yang Y, Qiu S, Qian L, Tian Y, Chen Y, Bi L and Chen W: OCF can repress tumor metastasis by inhibiting epithelial-mesenchymal transition involved in PTEN/PI3K/AKT pathway in lung cancer cells. PLoS One 12: e0174021, 2017.
19. Han M, Chen L and Wang Y: miR-218 overexpression suppresses tumorigenesis of papillary thyroid cancer via inactivation of PTEN/PI3K/AKT pathway by targeting Runx2. OncoTargets Ther 11: 6305-6316, 2018.

20. Chang M, Wu M and Li H: Curcumin combined with glycyrrhetinic acid inhibits the development of hepatocellular carcinoma cells by down-regulating the PTEN/PI3K/AKT signalling pathway. Am J Transl Res 9: 5567-5575, 2017.

21. Acosta SA, Tajiri N, Hoover J, Kaneko Y and Borlongan CV: Intravenous bone marrow stem cell grafts preferentially migrate to spleen and abrogate chronic inflammation in stroke. Stroke 46: 2616-2627, 2015.

This work is licensed under a Creative Commons Attribution-NonCommercial-NoDerivatives 4.0 International (CC BY-NC-ND 4.0) License. 